\title{
Simulating binocular visual field status in glaucoma
}

David P Crabb, Ananth C Viswanathan, Andrew I McNaught, Darmalingum Poinoosawmy, Frederick W Fitzke, Roger A Hitchings

\begin{abstract}
Aims-To simulate the central binocular visual field using results from merged left and right monocular Humphrey fields. To assess the agreement between the simulation and the binocular Humphrey Esterman visual field test (EVFT).

Method-59 consecutive patients with bilateral glaucoma each recorded Humphrey 24-2 fields for both eyes and binocular EVFT on the same visit. EVFT results were used to identify patients exhibiting at least one defect $(<10 \mathrm{~dB})$ within the central $20^{\circ}$ of the binocular field. This criterion is relevant to a patient's legal fitness to drive in the UK. Individual sensitivity values from monocular fields are merged to generate a simulated central binocular field. Results are displayed as a grey scale and as symbols representing defects at the $<10$ dB level. Agreement between patients failing the criterion using the simulation and the EVFT was evaluated.
\end{abstract}

Results-Substantial agreement was observed between the methods in classifying patients with at least one defect $(<10 \mathrm{~dB})$ within the central binocular field (kappa 0.81; SE 0.09). Patients failing this criterion using the EVFT results were identified by the binocular simulation with high levels of sensitivity $(100 \%)$ and specificity $(86 \%)$.

Conclusions-Excellent agreement exists between the simulated binocular results and EVFT in classifying glaucomatous patients with central binocular defects. A rapid estimate of a patient's central binocular field and visual functional capacity can be ascertained without extra perimetric examination.

Department of Visual Science, Institute of Ophthalmology, UCL, London

D P Crabb

F W Fitzke

Glaucoma Unit, Moorfields Eye Hospital, London A C Viswanathan A I McNaught

D Poinoosawmy

R A Hitchings

Correspondence to: David P Crabb, PhD, Department of Visual Science, Institute of Ophthalmology, University College London, Bath Street, London EC1V 9EL.

Accepted for publication 15 April 1998 field of vision that complies with the requirements for a legal fitness to drive.
Binocular visual field testing programs are readily available in various automated perimeters. However, additional binocular examination is not routinely administered in the clinical setting where perimetric resources are normally directed or "consumed" by monocular testing. Binocular visual field testing in glaucoma normally only becomes necessary when a patient presents with bilateral defects commonly seen in advanced stages of the disease and assessment of visual disability is required.

This paper describes a method of simulating a binocular visual field from the results of Humphrey monocular fields. This technique requires no extra perimetric testing and allows for a quick and useful estimate of a patient's central functional field. Purpose written computer software merges individual sensitivity values from left and right fields to generate a map of the central binocular visual field. Results are displayed as an easily interpretable grey scale with significant defects denoted by superimposed symbols. The purpose of the study was to assess the agreement between the results from this simulation and an automated test of the binocular field available on the Humphrey field analyser (Esterman visual field test, EVFT). Specifically, the study investigated the concordance between the techniques in classifying glaucomatous patients who exhibit defects with sensitivity values below 10 $\mathrm{dB}$ in the central binocular field. These type of field defects have a bearing on the Royal College of Ophthalmologists' recommendation for the legal fitness to drive in the UK. ${ }^{4}$

\section{Methods}

SUBJECTS AND DATA

Visual field data were acquired from 59 consecutive patients attending the glaucoma clinic at Moorfields Eye Hospital. All had previously presented with glaucomatous visual field loss in both eyes confirmed using the STATPAC 2 glaucoma hemifield test (GHT). ${ }^{56}$ In all cases GHT was outside normal limits. In addition, all patients had optic disc appearance in both eyes consistent with a clinical diagnosis of primary open angle glaucoma. All patients had no other ocular pathology, specifically cataract or macular degeneration, and all had previous experience of automated perimetry. Visual fields were recorded for both eyes with program 24-2 of the Humphrey field analyser (Humphrey Instruments, San Leandro, CA, USA) with a size III white stimulus in standard conditions using the full threshold strategy. Patients then underwent examination with the automated binocular EVFT which is available on the Humphrey field analyser. This test is 


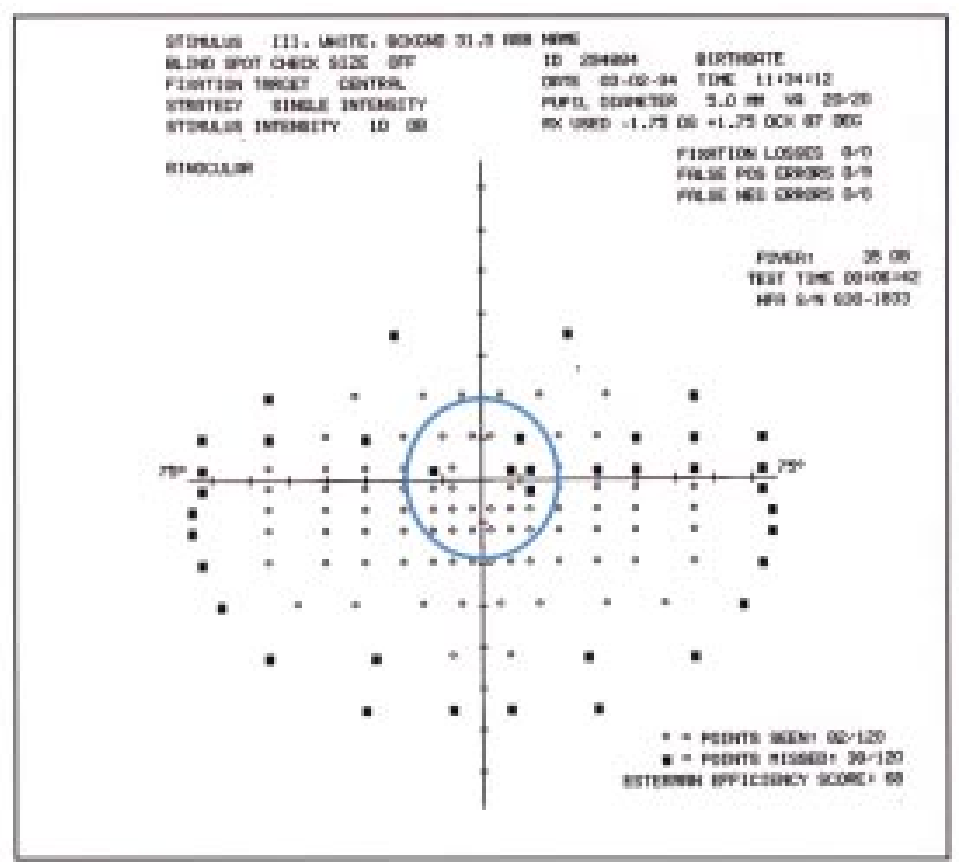

Figure 1 Esterman visual field test output from the Humphrey visual field analyser for one of the sample patients. A circle representing the central area $20^{\circ}$ from fixation has been superimposed. There are 24 test locations within the central area. This patient has five locations with sensitivity values below $10 \mathrm{~dB}$ within this area and is therefore classified as exhibiting "At least one defect $(<10 \mathrm{~dB})$ " using the EVFT analysis.

recognised by the International Perimetric Society and is recommended by the American Medical Association in its guide to visual impairment. ${ }^{3}$ In each case all field testing was completed during one visit. The EVFT was administered after the 24-2 examinations. The study was conducted during a routine hospital clinic and this precluded using a random sequence of field examinations but rest periods between tests were implemented throughout.

The binocular EVFT examines more than $130^{\circ}$ field and consists of a grid of 120 test points (Fig 1). The aim of this design is to produce a relative scale of defects based on function, with greater weighting in areas considered more useful to the patient. ${ }^{1}$ Each location is tested once with a size III white stimulus at an intensity of $10 \mathrm{~dB}$. Missed points are retested, with a second negative response resulting in a recorded defect. False positive and false negative responses are assessed in a similar fashion to the monocular programs. In the binocular mode the video eye monitor is aligned to the bridge of the nose, enabling central head positioning. Stability of fixation is monitored indirectly by observation since the automatic checking of fixation via the blind spot cannot be employed.

The visual field data were transferred to a personal computer and further analysed with purpose written software using s-PLUS 3.2 for Windows (StatSci Europe, MathSoft Inc, Oxford) and PROGRESSOR for Windows (Institute of Ophthalmology, London). Where a double determination of sensitivity had been undertaken at a given test location in the 24-2 program, the value of the sensitivity was taken as the mean of the two determinations.
The rationale for studying the central $20^{\circ}$ of the binocular visual field is related to the recommendation for the standard of the minimum field of vision for safe driving in the UK. The Royal College of Ophthalmologists in its advice to the Driving and Vehicle Licensing Authority (DVLA) of the UK has suggested:

".... a field of vision of at least $120^{\circ}$ on the horizontal measured by the Goldmann Perimeter using the III4e settings (or equivalent perimetry). In addition there should be no significant [field] defect in the binocular field which encroaches within $20^{\circ}$ of fixation either above or below the [horizontal] meridian... . Isolated scotomata represented in the binocular field near to the central fixation point may also be inconsistent with safe driving."

This standard is not equipment specific. The EVFT is one of the recommended "equivalent" examinations and is routinely used in cases of equivocal field loss. The advice to the DVLA does not, however, specify the number or configuration of EVFT defects that constitute a "significant" field defect in terms of legal fitness to drive.

This study is concerned only with recorded defects within the central $20^{\circ}$ of fixation. Twenty four of the EVFT locations fall within this area as highlighted in Figure 1. The purpose of this study was to identify patients who exhibited at least one defect $(<10 \mathrm{~dB})$ within the central $20^{\circ}$.

Therefore, patients were classified as having "At least one defect $(<10 \mathrm{~dB})$ " or "No defects $(<10 \mathrm{~dB})$ " using the EVFT.

\section{CENTRAL BINOCULAR FIELD SIMULATION}

Each location in the right monocular field has a corresponding point in the left monocular field in binocular viewing. The maximum sensitivity from each of the two overlapping locations was determined to give an estimate of sensitivity at that point, as if the subject was viewing in the binocular mode. These merged results can be displayed as a grid of sensitivity values or a grey scale. Individual locations with sensitivity values below $10 \mathrm{~dB}$ can be flagged by symbols. These can be considered equivalent to EVFT type defects. This process is described in the schematic diagram shown in Figure 2. Thirty two of the simulation locations fall within the central $20^{\circ}$ of fixation. The EVFT test pattern examines fewer locations (24 points) in the central $20^{\circ}$. Therefore, eight locations with no direct equivalent in the EVFT were excluded from the binocular simulation analysis. Four of these locations are sited in the superior hemifield and the remaining four are the closest to fixation (Humphrey coordinates plus or minus $3^{\circ}$ ). Patients were classified as having "At least one defect $(<10 \mathrm{~dB})$ " or "No defects $(<10 \mathrm{~dB})$ " using the binocular simulation.

All patients were classified in categories of the defect criterion for both the EVFT and binocular simulation analysis. Level of agreement between the two methods was evaluated using a kappa coefficient which gives an indication of agreement beyond chance. ${ }^{7}$ 


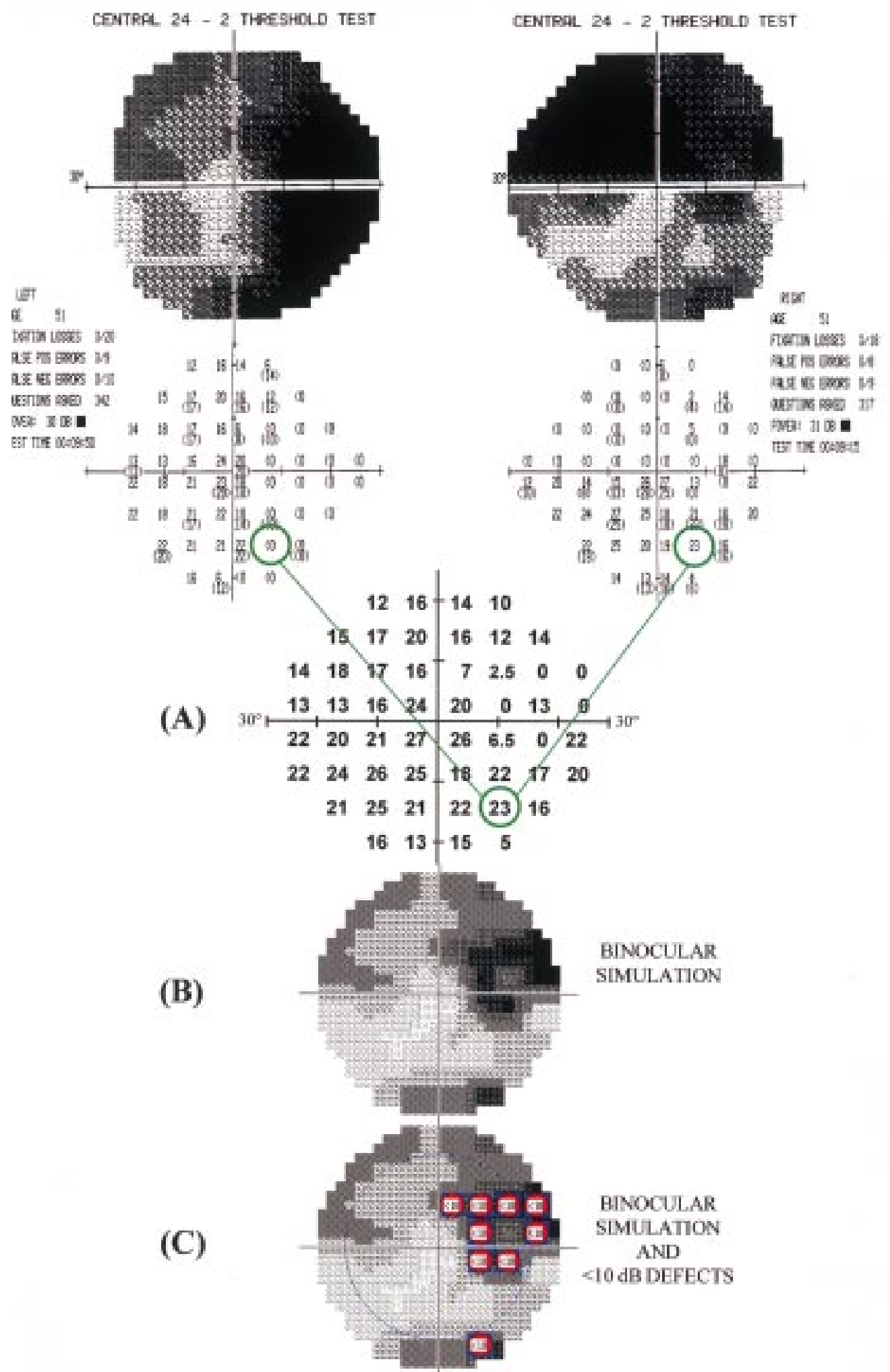

w

(⿸丆口

을

으

㞼

음

จ

œ

$\overrightarrow{0}$

$\overrightarrow{\vec{\omega}}$

흠

N

$\vec{\square}$

స్

윽

ż

$\frac{\widehat{D}}{3}$

음

$\overrightarrow{0}$
$\varnothing$

망

引

잉

음

굴

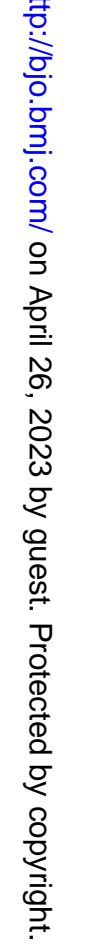

Figure 2 A schematic diagram describing the binocular simulation technique using data from the same patient shown in Figure 1. Humphrey 24-2 fields from the left and right eyes are merged point by point. The maximum sensitivity from each of the two corresponding points is determined to give an estimate of sensitivity at that point as if the subject was viewing in the binocular mode $(A)$. The resultant simulated central binocular field can be displayed as Humphrey type grey scale (B). The central area $\left(20^{\circ}\right.$ from fixation) can be highlighted and individual locations with sensitivity values below $10 \mathrm{~dB}$ are denoted by symbols $(C)$. Note the similarity of the locations exhibiting defects $(<10 \mathrm{~dB})$ compared with the EVFT output in Figure 1. 


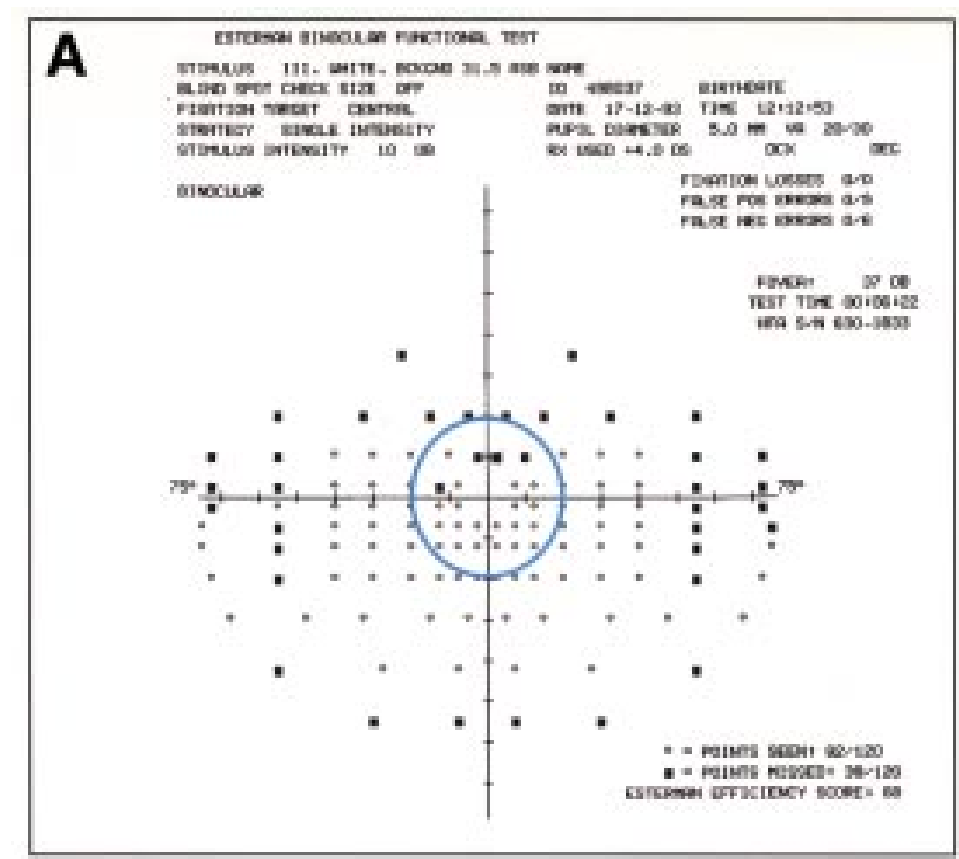

B
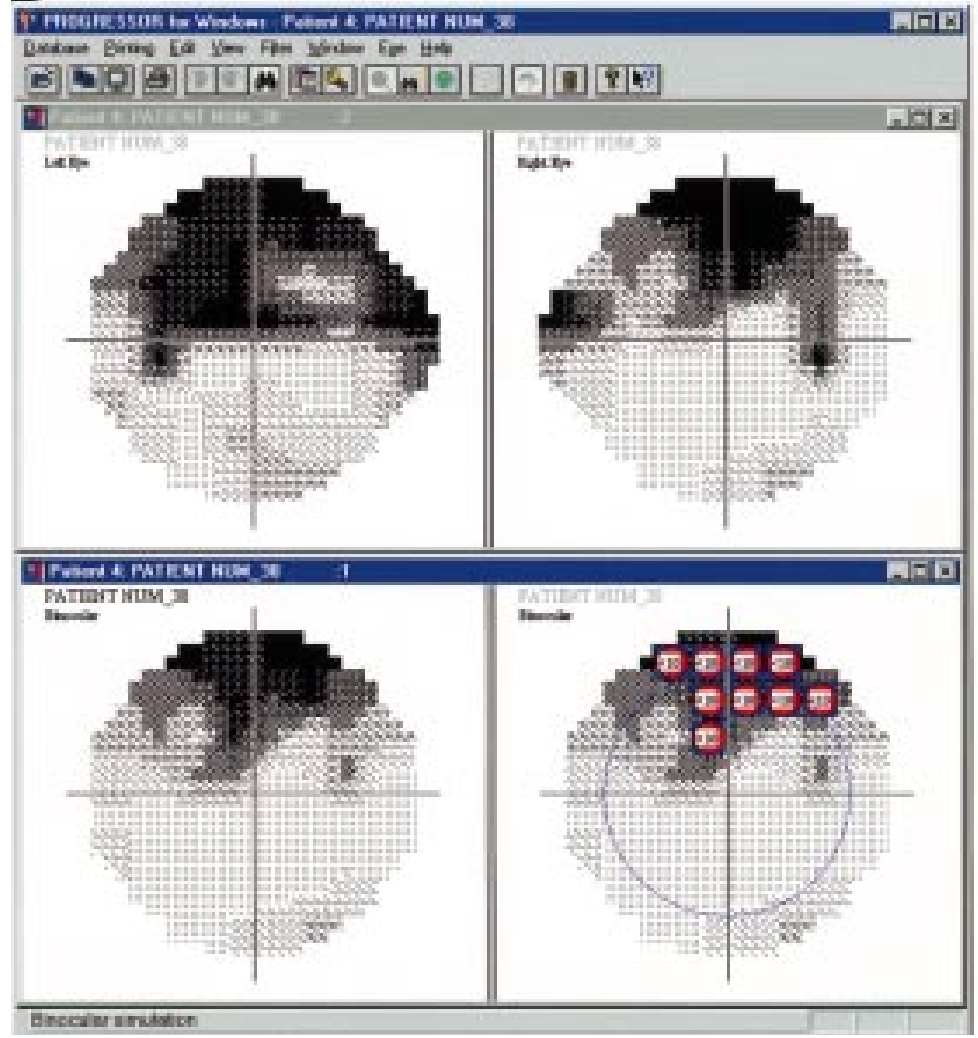

Figure 3 EVFT output for a sample patient is shown in $(A)$. A circle has been superimposed to denote the central $20^{\circ}$. Results from the binocular simulation implemented by the PROGRESSOR for Windows software (Institute of Ophthalmology, London) are shown for the same patient in (B). The sensitivity of the left and right 24-2 fields are shown as Humphrey type grey scales. The lower panels show the results from the binocular simulation as Humphrey type grey scale with and without superimposed symbols denoting point by point EVFT type defects $(<10 \mathrm{~dB})$. The blue circle on the display indicates the central $20^{\circ}$ area. This patient clearly demonstrates "At least one defect $(<10 \mathrm{~dB})$ " using the EVFT (A) and similarly "At least one defect $(<10 \mathrm{~dB})$ " using the binocular simulation (B).

\section{Results}

The mean age of the patients at examination was 65.5 years (range $30-85$ years). Patients presented with a wide range of visual field defects in both eyes. The sample mean
Table 1 Number of patients classified as having at least one defect or no defects $(<10 \mathrm{~dB})$ in the central field by EVFT analysis and binocular simulation

\begin{tabular}{llll}
\hline Binocular simulation & $\begin{array}{l}\text { At least one defect } \\
(<10 \mathrm{~dB})\end{array}$ & $\begin{array}{l}\text { No defects } \\
(<10 \mathrm{~dB})\end{array}$ & Total \\
\hline $\begin{array}{l}\text { At least one defect } \\
\quad(<10 \mathrm{~dB})\end{array}$ & 15 & 4 & 19 \\
$\begin{array}{l}\text { No defects }(<10 \mathrm{~dB}) \\
\text { Total }\end{array}$ & 0 & 25 & 25 \\
\hline
\end{tabular}

Humphrey MD (mean deviation) was -11.5 (SD 8.2) $\mathrm{dB}$ for the right eye and -10.6 (7.7) $\mathrm{dB}$ for the left eye. The sample mean Humphrey CPSD (corrected pattern standard deviation) was 7.1 (3.7) $\mathrm{dB}$ for the right eye and 7.0 (3.8) $\mathrm{dB}$ for the left eye.

The adequacy of perimetric performance was assessed using the Humphrey reliability indices. ${ }^{8}$ A visual field was considered unreliable if $33 \%$ or more was recorded for at least one of the false positive (FP), false negative (FN), and fixation loss (FL) indices. These limits have been previously recommended for identifying inadequate perimetric performance. ${ }^{9}{ }^{10}$ Patients were excluded from further analysis if at least one field examined on the 24-2 test or the EVFT was unreliable (FP and $\mathrm{FN}$ are also available on the EVFT). In total, 15 patients were excluded from further analysis because of inadequate perimetric performance in at least one of the tests. (Eleven patients were unreliable on at least one monocular field; two unreliable on EVFT; two unreliable on at least one monocular field and EVFT.)

Results from EVFT and binocular simulation analysis for example patients are shown in Figures 3 and 4. Classification by the EVFT and binocular simulation analysis of the reliable 44 patients as exhibiting "At least one defect $(<10 \mathrm{~dB})$ " or "No defects $(<10 \mathrm{~dB})$ " in the central field $\left(20^{\circ}\right.$ from fixation) is shown in Table 1. The kappa coefficient of agreement was 0.81 (SE 0.09). This value indicates a substantial level of agreement between the two techniques. ${ }^{11}$ All patients that exhibited "At least one defect $(<10 \mathrm{~dB})$ " using the EVFT were correctly identified by the binocular simulation (100\% sensitivity). Four of the 29 patients defined as having "No defects $(<10$ $\mathrm{dB})$ " using the EVFT were classified by the binocular simulation as having a defect $(86 \%$ specificity).

\section{Discussion}

This study has illustrated a new method of simulating the central binocular field by merging results from monocular Humphrey fields. Using the higher sensitivity from either eye for each test location has been shown to be superior to other methods in combining monocular results to estimate a binocular field in glaucoma patients. ${ }^{12}$ Earlier studies, using results from Goldmann perimetry, have considered this concept but concluded that ideally a patient's functional field capacity should only be accurately assessed via binocular viewing because of what is termed enhancement or sensitivity summation. ${ }^{1}$ Binocular enhancement occurs in areas where the two monocular fields overlap and has the effect of increasing 


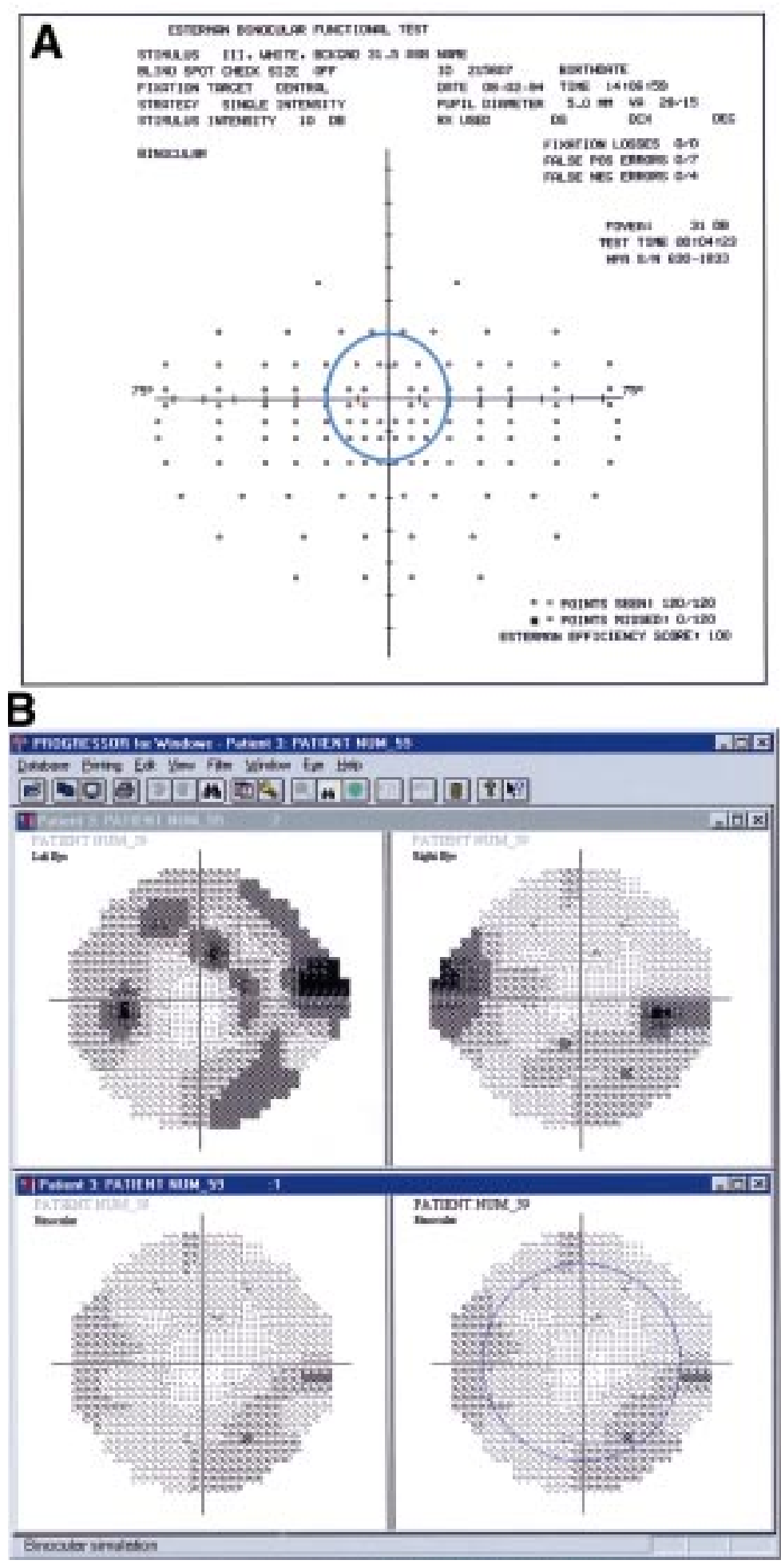

Figure 4 EVFT output $(A)$ and results from the binocular simulation (B) implemented by the PROGRESSOR for Windows software (Institute of Ophthalmology, London) are shown for another sample patient. The lower panels show the results from the binocular simulation as Humphrey type grey scale with and without superimposed symbols denoting point by point EVFT type defects $(<10 \mathrm{~dB})$. This patient demonstrates "No defects" using the EVFT (A). This patient also demonstrates "No defects" using the binocular simulation (B) despite the presence of areas of visual field damage in each monocular field.

the Esterman score (percentage of 120 EVFT locations where the stimulus was seen) above that predicted by merging the two monocular fields. Enhancement has been demonstrated in normal subjects. ${ }^{13}$ However, there is evidence that it occurs less often in patients with advanced glaucoma. ${ }^{2} 1415$ The results from our study also suggest that enhancement occurs infrequently in the central field $\left(20^{\circ}\right.$ from fixation) of a group of patients with bilateral glaucoma. Significant binocular enhancement would have manifested poor agreement between the simulation and the EVFT in identifying patients with at least one defect $(<10$ $\mathrm{dB}$ ). This was not the case since high levels of agreement were observed.

A recent study, comparing Esterman score against mean deviation of monocular fields, reported lower than expected number of EVFT defects in glaucoma patients with bilateral field loss. ${ }^{16}$ Moreover, the automated binocular EVFT has been described by others as "less stringent" and "more forgiving" than other perimetric tests. ${ }^{4}$ These findings may of course be explained in part by binocular enhancement but other explanatory factors are likely to be more significant. For example, the test strategy employed by the EVFT on the Humphrey field analyser is not optimal. Retesting only a selected sample of test locations where the stimulus was not seen provides a simple evaluation of the binocular field. However, in the presence of the inherent variability in patient response to the light stimulus, this test strategy is biased. ${ }^{17}$ Results from a study investigating the performance of this type of test strategy indicated that the true number of binocular defects can be underestimated by approximately $20 \% .{ }^{17}$

There is no direct monitoring of fixation on the Humphrey EVFT. In contrast, the binocular simulation is based on results from the Humphrey monocular fields where fixation is monitored throughout the test by a method that presents stimuli at the site of the predetermined blind spot. Control of fixation is an important component of perimetric testing and reliability of response. ${ }^{18}$ While the lack of fixation monitoring on the EVFT may mimic a "real" field of view it may also inflate the number of points seen compared with results from merging the monocular fields.

The obvious limitation of the binocular simulation is that it can only provide an assessment of the central field whereas the EVFT tests the full peripheral field across $130^{\circ}$. It has, however, been suggested that the distribution of points on the Esterman grid does not fully reflect the claimed functional significance. ${ }^{19}$ For example, it has been shown that the central $10^{\circ}$ may provide the input for up to half of the primary visual cortex. ${ }^{20}$ It may be that a functional assessment based on central areas may have a wider role in assessing visual ability.

The binocular simulation may provide an indication that a patient with bilateral defects should be further investigated in terms of legal fitness to drive. This is particularly relevant in the UK where currently the recommended definition for the standard of the minimum field of vision for safe driving includes, “... no significant [field] defect in the binocular field which encroaches within $20^{\circ}$ of fixation [either] above or below the [horizontal] meridian." ${ }^{4}$ The high level of sensitivity $(100 \%)$ and specificity (86\%) associated with 
the binocular simulation in determining whether a glaucomatous patient with bilateral defects will have at least one defect $(<10 \mathrm{~dB})$ on the EVFT suggests this may be a useful guide for clinicians. Patients demonstrating defects on the binocular simulation are likely to exhibit defects in the central area of the EVFT and may warrant further investigation. Equally, patients presenting with no defects on the binocular simulation are likely to exhibit no defects in the central area of the EVFT. The utility of this indicator that a glaucomatous patient may fail the criteria for legal fitness to drive in the UK may be proved by establishing a link between defects in the central field and significant defects in the peripheral field. An investigation is under way but actual testing of the peripheral binocular field is likely to remain central to assessing legal fitness to drive. Nevertheless, the simulation provides the clinician with a rapid and routine assessment of the central binocular field. This may be particularly useful since it has been suggested that alerting elderly drivers to deterioration in their visual performance, so that they can make compensatory changes in their driving behaviour, may be important. ${ }^{21}$

A recent study demonstrated that while glaucoma is a leading cause of visual disability, in many cases visual field progression is not sufficient to lead to bilateral blindness. ${ }^{22}$ The study concluded that glaucoma management should be closely tailored to the confirmed rate of visual field loss in an individual. A framework for the analysis of the rate of field loss at individual test locations has been developed..$^{23}$ By using this rate of change in a given series of fields it is possible to predict the sensitivity at subsequent fields. Used in tandem with the binocular simulation described in this study it may be possible to predict the future status of a patient's central binocular field. These predictions of future changes to the functional field may prove to be useful in the management of glaucoma and demonstrate the "relevance" of the insidious behaviour of glaucomatous progression to individual patients. Such forecasts may be impeded by the inherent variability that exists between visual field results $^{25}$ but improved perimetric test strategies $^{2627}$ and variability reduction techniques ${ }^{28}$ may reduce this limitation.

In summary, a new method for simulating binocular visual field status in glaucoma by merging results from Humphrey monocular fields has been demonstrated. Results are computed quickly, and displayed in an easy to interpret manner on a computer screen. Substantial agreement exists between the simulated binocular results and EVFT in classifying glaucomatous patients with central binocular defects. In contrast with the EVFT, results from the simulation are based on sensitivity values from a full threshold test strategy and are controlled for fixation. The simulation cannot currently estimate the status of the binocular field beyond the central area. However, it may be a clinically useful indicator for deter- mining whether a patient is likely to fulfil the visual field requirement for legal fitness to drive in the UK. In conclusion, this new method provides a rapid estimate of a patient's central binocular field and visual functional capacity. This can be ascertained in a routine clinical environment without the need for extra perimetric examination. This work is funded in part by grants from the Royal National
Institute for the Blind, the International Glaucoma Association, anstitute for the Bedical Research Council. The authors thank David Edgar (Applied Vision Research Centre, City University, Edgar (Applied Vision Research Centre, City
London) for helpful comments on the manuscript.

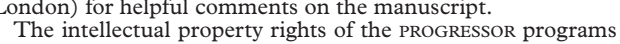
belong to the Institute of Ophthalmology and Moorfields Eye Hospital of which the authors are employees.

1 Esterman B. Functional scoring of the binocular field. Ophthalmology 1982;89:1226-34.

2 Mills RP, Drance SM. Esterman disability rating in severe glaucoma. Ophthalmology 1986;93:371-8.

3 American Medical Association. Guides to the evaluation of permanent impairment. 3rd ed. Chicago: AMA, 1988.

permanent impairment. 3rd ed. Chicago: AMA, 1988 .
Taylor JF. Medical aspects of fitness to drive. A guide for medical practitioners. 5th ed. London: HMSO, 1995:119-21.

5 Aractitioners. 5th ed. London: HMSO, 1995:119-21. visual field evaluation. Arch Ophthalmol 1992;110:812-9.

6 Katz J, Quigley HA, Sommer A. Repeatability of the glaucoma hemifield test in automated perimetry. Invest Ophthalmol Vis Sci 1995;36:1658-64.

7 Fleiss JL. Statistical methods for rates and proportions. New York: John Wiley, 1981:212-25.

8 Heijl A, Lindgren G, Olsson J. A package for the statistical analysis of visual fields. Doc Ophthalmol Proc Ser 1987;49: 153-68

9 Katz J, Sommer A. Reliability parameters of automated perimetric tests. Arch Ophthalmol 1988;106:1252-4.

10 Bickler-Bluth M, Trick GL, Kolker AE, et al. Assessing the utility of reliability indices for automated visual fields. $O p h-$ thalmology 1989;96:616-9.

11 Landis JR, Koch GG. The measurement of observer agreement for categorical data. Biometrics 1977;33:159-74

12 Nelson-Quigg JM, Johnson CA, Cello KE. Predicting Nelson-Quigg JM, Johnson CA, Cello KE. Predicting
binocular visual field sensitivity of glaucoma patients using monocular visual field data. (ARVO abstracts.) Invest Ophthalmol Vis Sci 1997;38(suppl):2645.

13 Wood JM, Collins MJ, Carkeet A. Regional variations in binocular summation across the visual field. Ophthalmic Physiol Opt 1992;12:46-51.

14 Calabria G, Capris P, Burtolo C. Investigations on space behaviour of glaucomatous people with extensive visual field loss. Doc Ophthalmol Proc Ser 1983;35:201-10.

15 Calabria G, Gandolfo E, Rolando M, et al. Ergoperimetry in patients with severe visual field damage. Doc Ophthalmol Proc Ser 1985;42:537-47.

16 Harris ML, Jacobs, NA. Is the Esterman binocular field sensitive enough? In: Mills RP, Wall M, eds. Perimetry update 1994/1995. Amsterdam: Kugler, 1995:13-24.

17 Smith TL. The effect of regression towards the mean on visual disability ratings. Doc Ophthalmol 1989; 53:343-80.

18 Henson DB, Evans J, Chauhan BC, et al. Influence of fixation accuracy on threshold variability in patients with open angle glaucoma. Invest Ophthalmol Vis Sci 1996;37:444-50.

19 Colenbrander A, Lieberman MF, Schainolz DC. Preliminary implementation of the functional vision score system on the Humphrey Field Analyser. In: Mills RP, ed. Perimetry update 1992/1993. Amsterdam: Kugler, 1993: 487-96.

20 Wässle $\mathrm{H}$, Grunert $\mathrm{U}$, Rohrenbeck J, et al. Retinal ganglion cell density and cortical magnification factor in the primate. Vision Res 1990;30:1897-911.

21 Charman WN. Vision and driving - a literature review and commentary. Ophthalmic Physiol Opt 1997;17:371-91.

22 Quigley HA, Tielsch JM, Katz J, et al. Rate of progression in open-angle glaucoma estimated from cross-sectional prevalence of visual field damage. Am $\mathcal{f}$ Ophthalmol 1996;122: lence of

23 McNaught AI, Crabb DP, Fitzke FW, et al. Modelling series of visual fields to detect progression in normal-tension glaucoma. Graefes Arch Clin Exp Ophthalmol 1995;233: $750-5$.

24 Fitzke FW, Hitchings RA, Poinoosawmy D, et al. Analysis of visual field progression in glaucoma. Br F Ophthalmol 1996; 80:40-8.

25 O'Brien C, Wild JM. Automated perimetry in glaucomaroom for improvement. Br f Ophthalmol 1995;79:200-1.

26 Olsson J, Rootzén H. An image model for quantal response analysis in perimetry. Scand F Statist 1994;21:373-87.

27 Heiil A. Improved test algorithms for automated perimetry. In: Krieglstein GK, ed. Glaucoma update V. Heidelberg: Kaden Verlag, 1995:168-73.

28 Fitzke FW, Crabb DP, McNaught AI, et al. Image processing of computerised visual field data. $\mathrm{Br} \mathcal{F}$ Ophthalmol 1995;79:207-12. 